\title{
Dispersion Suppression in the MPRI Achromat Beam Line
}

\author{
V.A. Anferov, M. Ball, D.L. Friesel, W.P. Jones, \\ Indiana University Cyclotron Facility, Bloomington, IN 47408
}

\section{Abstract}

The first section of the beam transport system for Midwest Proton Radiation Institute (MPRI) [1] has been constructed. It is designed to compensate both spatial and angular dispersion of $205 \mathrm{MeV}$ proton beam coming out of the Indiana University Cyclotron [2]. We present data obtained during the commissioning of the beam line including measurements of the beam dispersion and the beam emittance out of the cyclotron.

\section{INTRODUCTION}

The newly constructed achromat beam line is the first section of the $205 \mathrm{MeV}$ proton beam transport system for the MPRI. Its flexible optics design [3] is based on a pair of opposed $24^{\circ}$ dipoles, which allows one to tune the beam line for compensation of both spatial and angular dispersion of the $205 \mathrm{MeV}$ beam coming out of the Indiana University Cyclotron. This ion-optical condition would eliminate beam position fluctuations caused by momentum changes of the cyclotron beam.

The layout of the beam line is shown in Fig. 1. The $205 \mathrm{MeV}$ protons first go through 5.5-m-long straight section which contains 3 harps for beam emittance measurements and time-of-flight system for energy measurements. The five quadrupoles of the straight section provide optical matching between the cyclotron and the dispersion compensating dogleg. The dogleg consists of two opposite $24^{\circ}$ bends with four quadrupoles between the dipoles. The following $45^{\circ}$ bending magnet brings the beam to the direction of the trunk line which would feed the beam into the two planned treatment rooms.

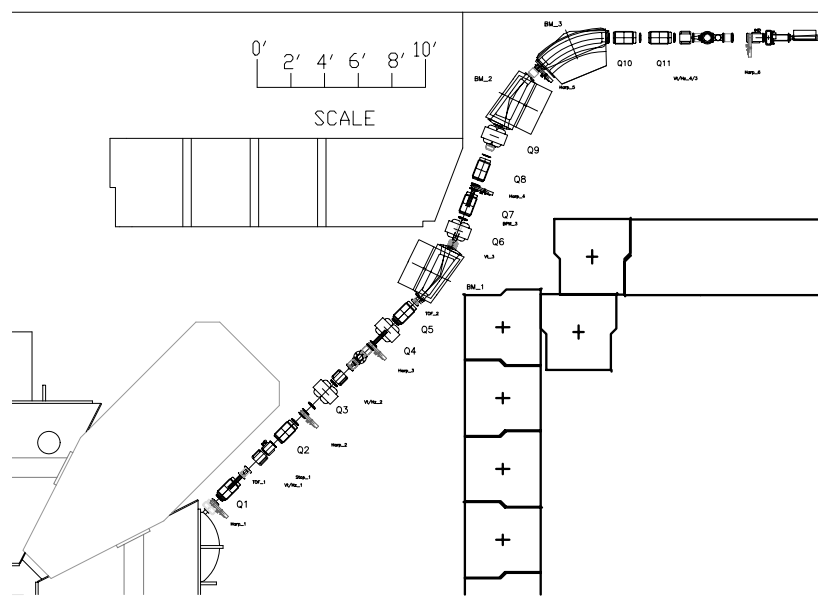

Figure 1: Layout of the MPRI achromatic beam line.

\section{DISPERSION MEASUREMENTS}

An ideal separate-sector-cyclotron has zero angular dispersion while its spatial disperson is proportional to the bending radius:

$$
D_{x}=\frac{\rho}{1-\tan \delta}
$$

where $\delta=27^{\circ}$ is the edge angle of the IU cyclotron sector magnets. However, injection conditions may result in a mismatch between the center of the orbits and the geometrical/magnetic center of the cyclotron. This in turn would change the value of spatial dispersion but more importantly would give rise to angular dispersion. Thus, it is important to measure the dispersion out of the cyclotron.

An attractive idea to measure the dispersion in a beam line is to scale the current in all magnets by $1 \%$ and observe the beam position variation at the end of the beam line. Indeed, scaling the current in beam line magnets is equivalent to changing the beam momentum. However, in order to measure dispersion at the end of the beam line one should also change the initial beam position and angle according to the initial dispersion values, which are unknown. Otherwise, this technique would measure the dispersion of the beam line itself and may mislead to a conclusion that the dispersion at the exit of the cyclotron is zero.

There is also a potential method of deriving the dispersion from beam emittance measurements at two places where the dispersion is expected to be different. Unfortunately, the dispersion contribution to the beam emittance is usually small and hard to separate from the measurement's systematic errors.

The only remaining option for measuring the dispersion is to induce a small energy change of the extracted beam without changing the optics of the accelerator. We varied the energy by adjusting the rf-acceleration voltage to extract a different turn out of the cyclotron without changing the cyclotron's magnetic field. Using the time-of-flight system we measured the resulting energy change to be $220 \pm 20 \mathrm{keV}$, which corresponds to the momentum change $\Delta p / p=5.9 \cdot 10^{-4}$ at $205 \mathrm{MeV}$ energy of the extracted beam. We then measured the beam position change on Harp- 1 at the exit of the cyclotron and on Harp-3, which is $4.049 \mathrm{~m}$ downstream from Harp-1. All quadrupoles between the harps were turned off. The results are shown in Fig. 2. Fitting the beam profiles with gaussian curves, we determined the centroid for each profile. The results are summarized in Table 1.

Tracing back the obtained dispersion values through the cyclotron extraction optics, one would find that the dispersion at the extraction orbit was $D_{x}=-1.65 \mathrm{~m} D_{x}^{\prime}=0.68$. These values are different from the ideal-case prediction given by Eq. 1, which suggests $D_{x}=-2.77 \mathrm{~m}$ and 
Table 1: Dispersion measurements

\begin{tabular}{|l|c|c|}
\hline & Harp-1 & Harp-3 \\
\hline$E_{0}$ position & $-3.36 \pm 0.03 \mathrm{~mm}$ & $0.23 \pm 0.04 \mathrm{~mm}$ \\
$E_{0}-0.22 \mathrm{MeV}$ & $-2.42 \pm 0.03 \mathrm{~mm}$ & $4.67 \pm 0.01 \mathrm{~mm}$ \\
\hline$D_{x}(\mathrm{~m})$ & $1.59 \pm 0.15 \mathrm{~m}$ & $7.5 \pm 0.6 \mathrm{~m}$ \\
\hline$D_{x}^{\prime}$ & \multicolumn{2}{|c|}{$1.47 \pm 0.15$} \\
\hline
\end{tabular}

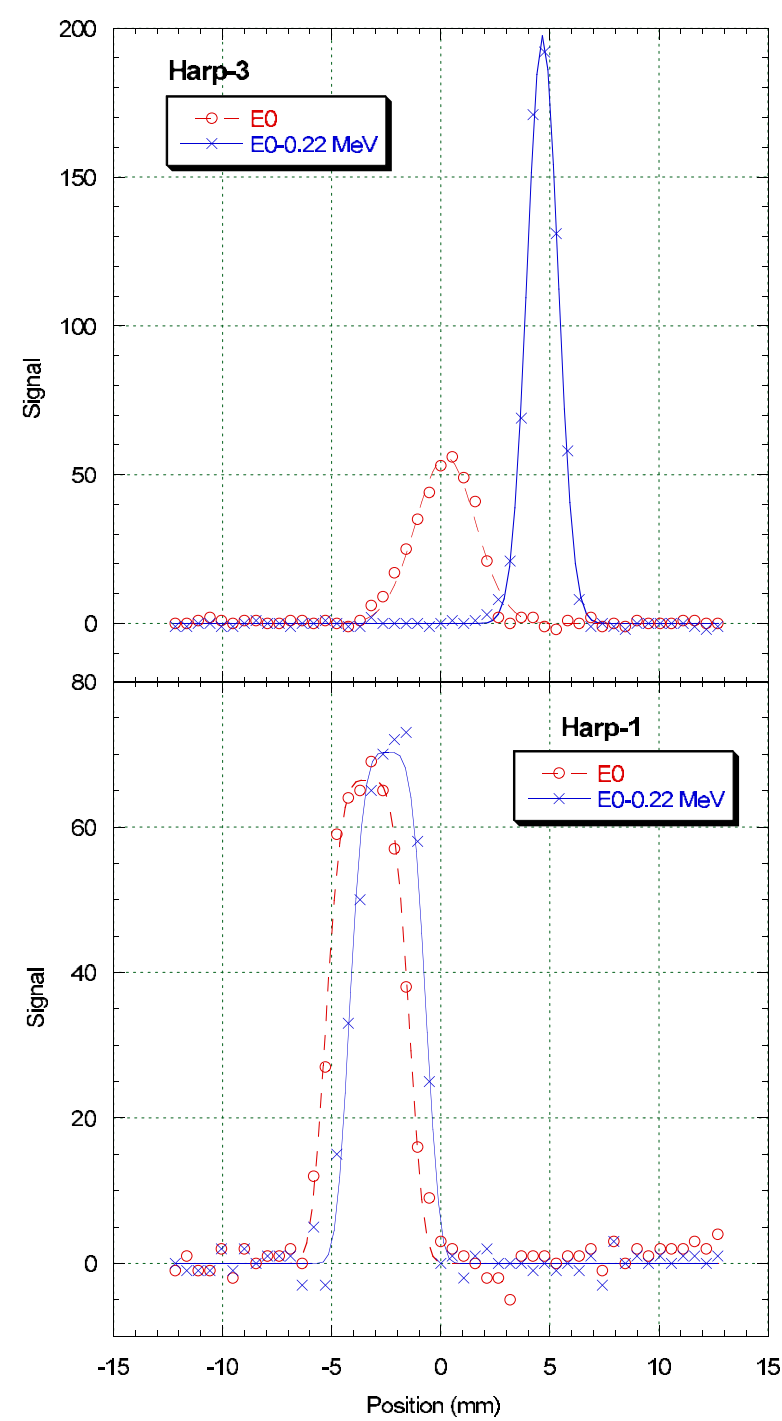

Figure 2: Horizontal beam profiles on Harps 1 and 3 for slightly different energies of the extracted beam. The curves are gaussian fits to the data; centroid of each beam profile is determined with these fits.

$D_{x}^{\prime}=0$. This indicates that there is a mismatch between the orbit center and the geometrical center of the cyclotron.

\section{EMITTANCE MEASUREMENT.}

Three harps in the first section of the beam line provide quick and easy way of measuring the transverse beam emit- tance [4]. Fitting the harp data with gaussian curves we determined the rms beam radius at each harp location. The results are shown in Table 2.

Before calculating the beam emittance and optical functions, one has to correct horizontal emittance data for the dispersion contribution:

$$
\frac{\epsilon \beta_{x}}{\pi}=\sigma^{2}-(D \cdot \delta)^{2}
$$

where $\delta=1.34 \cdot 10^{-4}$ is rms momentums spread in the beam. Table 3 shows calculated values of the optical functions and beam emittance at the location of Harp- 1 . The beam emittance images in the phase space are shown in Fig. 3. Note that we quote $2 \sigma$ beam emittance which contains $87 \%$ of the particles.

Table 2: RMS beam radius $\sigma$ at three harp locations.

\begin{tabular}{|c|c|c|}
\hline & Horizontal & Vertical \\
\hline Harp-1 & $1.275 \pm 0.04 \mathrm{~mm}$ & $0.34 \pm 0.005 \mathrm{~mm}$ \\
Harp-2 & $0.926 \pm 0.04 \mathrm{~mm}$ & $0.89 \pm 0.04 \mathrm{~mm}$ \\
Harp-3 & $1.28 \pm 0.03 \mathrm{~mm}$ & $1.75 \pm 0.06 \mathrm{~mm}$ \\
\hline
\end{tabular}

Table 3: Beam emittance and optical functions at the location of Harp-1. The values in "Horz.0" column were calculated neglecting the dispersion contribution, while column "Horz.1" includes the dispersion correction.

\begin{tabular}{|l|c|c|c|}
\hline & Horz.0 & Horz.1 & Vert \\
\hline$\beta(\mathrm{m})$ & 4.029 & 6.573 & 1.232 \\
$\alpha$ & 0.962 & 1.794 & 1.540 \\
$\epsilon(\pi \mu \mathrm{m} \cdot \mathrm{rad})$ & 1.612 & 0.962 & 0.364 \\
\hline
\end{tabular}

\section{ACHROMATIC SETTINGS FOR THE BEAM LINE}

Once both the dispersion and optical functions of the beam are determined at the entrance to the beam line, one can calculate the beam line settings to compensate the dispersion. We used MAD [5] code for calculating beam line settings for achromat properties. Both angular and spatial dispersion are brought to zero at the exit of 45 degree bending dipole. The beam envelopes in vertical and horizontal plane are plotted in Fig. 4. Quadrupole settings for achromatic beam line are listed in Table 5. Note that the last two quadrupoles after the 45 degree bend are only used for matching the beam optics between the achromat and the subsequent trunk line, which will be constructed during the summer of 2001.

\section{CONCLUSIONS}

The first section of the beam line for Midwest Proton Radiation Institute has been assembled and studied. In its 


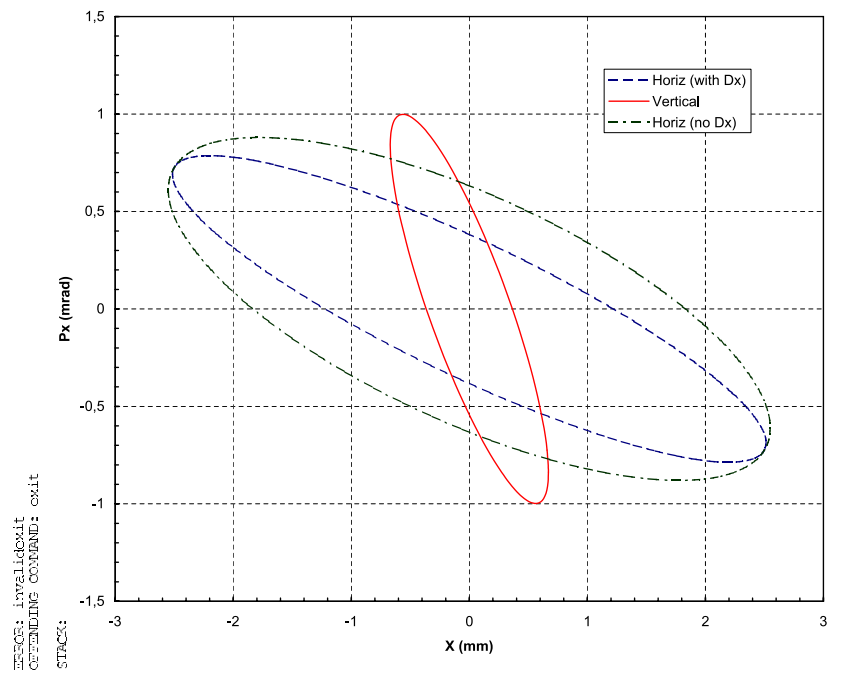

Figure 3: Horizontal and vertical beam emittance at the exit from the cyclotron. The "dash-dot" curve corresponds to horizontal beam emittance calculated neglecting dispersion effect in the beam line.

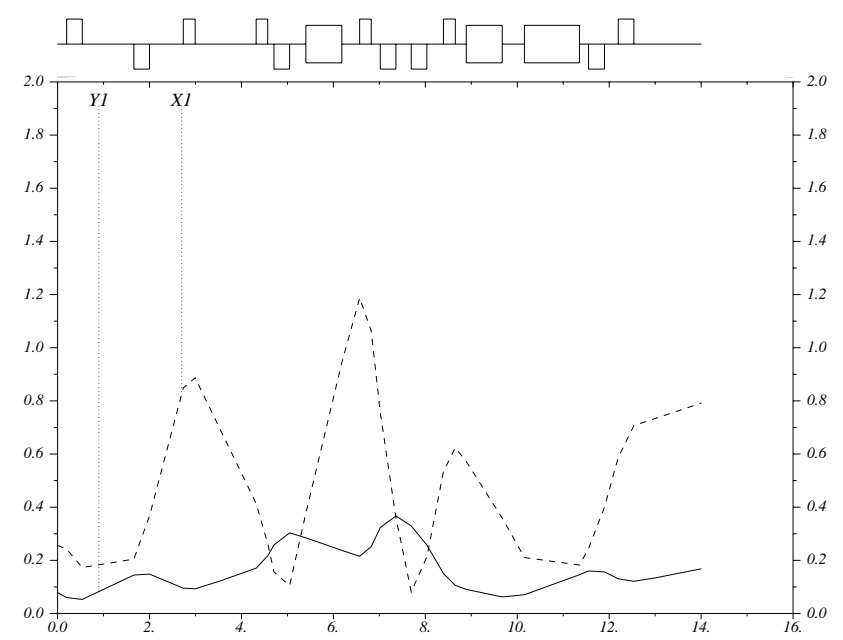

Figure 4: Horizontal and vertical beam envelopes in the achromatic beam line. Both angular and spatial dispersion are zero at the exit of the 45 degree bending magnet.
Table 4: Calculated quadrupole settings for achromatic beam line. Quadrupole strength is given in energy independent units of Gradient/B $\rho$. Horizontally focusing quads have positive strength.

\begin{tabular}{|l|c|}
\hline Name & Strength $\left(m^{-2}\right)$ \\
\hline Quad 1 & 3.5770 \\
Quad 2 & -3.4168 \\
Quad 3 & 4.4759 \\
Quad 4 & 5.0364 \\
Quad 5 & -3.6233 \\
Quad 6 & 7.3940 \\
Quad 7 & -3.9115 \\
Quad 8 & -1.7838 \\
Quad 9 & 7.2153 \\
\hline
\end{tabular}

present setup it has been used for radiation research experiments. Excellent beam transmission and reproducibility has been achieved in the beam line. During the commissioning period we measured both the beam emittance and dispersion at the exit of the cyclotron. Settings of the beam line quadrupoles were calculated to compensate the dispersion out of the cyclotron. We plan to test achromatic properties of the beam line in the fall of 2001 after the summer cyclotron shutdown.

\section{REFERENCES}

[1] V. Anferov et al., "The Indiana University Midwest Proton Radiation Instutute", these Conf. Proc.

[2] R.E. Pollock, PAC'77, IEEE Trans. Nucl. Sci, Vol. NS-24, 3, 1505 (1977).

[3] W.P. Jones, G.P.A. Berg, in Proc. of PAC-99, New York, 2519 (1999).

[4] S.Y. Lee, "Accelerator Physics", p.56, World Scientific, 1999.

[5] H. Grote, F.C. Iselin, "Methodical Accelerator Design", CERN/SL/90-13 (1996). 